\title{
PHASE COMPOSITION, TEXTURE AND CURRENT-CARRYING ABILITY OF Ag-HTSC-Ag THREE-LAYER CONDUCTORS
}

\author{
YU. PERLOVICH*, M. ISAENKOVA*, A. SHIKOV**, \\ N. KALININ** and T. GORSHKOV** \\ *Moscow Engineering Physics Institute, Kashirskoe Shosse 31, \\ 115409 Moscow, Russia \\ **All-Russia Scientific-Research Institute of Inorganic Materials, \\ ul.Rogova, 123060 Moscow, Russia
}

(Received 10 October 1994; in final form 20 November 1994)

\begin{abstract}
The regularities of phase changes and texture formation in composite superconductors Ag-HTSC-Ag, produced by electrophoretical deposition, are studied. Depending on phase composition of the initial ceramic powder, cyclic treatment of the composites, including pressing and annealing, results in different development of the basal texture, being necessary for attainment of high critical current density.

The basal texture forms primarily in the phase 2212 , which prevailed in the initial ceramic powder and transforms gradually into the phase 2223 during heat treatment. The derivative phase 2223 , being of practical interest, shows either heritage or scattering of the basal texture in accord with the liquid phase content in the sample under annealing. Mechanisms of texture development in ceramic phases were proposed.

A diagram for composites Ag-HTSC-Ag, connecting their phase composition and texture perfection with measured critical current density, is constructed.
\end{abstract}

KEY WORDS: Bismuth ceramics, superconductor, phase composition, texture, critical current density.

\section{INTRODUCTION}

The anisotropy of properties of HTSC-ceramics, being caused by the crystalline lattice structure, determines the importance of crystallographic texture for attainment of the high current-carrying ability of superconductors. The sharper the distribution of basal normals, the higher is the value of the critical current in the plane perpendicular to the axis of their preferential orientation.

At the same time superconductivity is influenced also by the character of intergranular boundaries which correlates with the texture perfection. Since the probability to meet a high-angle boundary decreases as grains tend to be oriented in the same manner, the texture reflects indirectly misorientation of neighbouring grains in the analyzed specimen. Though the main technological trend in HTSC production is to seek for the sharpest crystallographic texture, it should be taken into account that points of pinning can settle at boundaries and therefore some content of (sub)boundaries with optimal parameters ought to be retained in a superconducting specimen. If this is really so, a definite texture scattering would be necessary for attainment of maximal properties. 
In order to control the texture of HTSC-ceramics we need to know the regularities of its development under pressing, rolling and heat treatment, i.e. during typical technological procedures being used for conductor manufacturing. But texture development in a HTSC-ceramic is inseparably connected with its phase composition at successive stages of processing. Therefore an effective study of texture formation as applied to HTSC-ceramics is possible only within the frame-work of complex X-ray investigation including phase analysis, also.

Earlier, in the work (Perlovich et al., 1992) the processes of structure and texture formation in electrophoretical coatings of bismuth ceramics $\mathrm{Bi}_{4} \mathrm{Sr}_{3} \mathrm{Ca}_{3} \mathrm{Cu}_{4} \mathrm{O}_{x}$ were studied. Possibilities were considered for the improvement of the texture in these coatings by use of different heat treatments. In the deposited textureless layer, consisting predominantly of the phase 2212 , the compacting electro-impulse compression produces initially a relatively weak basal texture, while the subsequent heat treatment at $840^{\circ}-870^{\circ} \mathrm{C}$, causing partial melting of the ceramic layer, creates in this layer a very sharp texture of the same type. Its development is accompanied by formation of the secondary phase 2201, which does not participate in "high-temperature" superconductivity, though some optimal density of interphase boundaries in the specimen seems to be favourable for attainment of satisfactory superconducting properties.

The mechanism responsible for texture development during heat treatment was analyzed. It includes partial melting of the ceramic layer, the following alignment of residual solid-phase plate-like fragments in the field of surface-tension forces with the plane (001) parallel to the melt surface and crystallization of the melt while these fragments are in the role of nucleation centers.

The present study, being a continuation of the work (Perlovich et al., 1992) deals with three-layer conductors Ag-HTSC-Ag which are the prototype of a long-size wire for practical usage. However, unlike the previous work, here the bismuth ceramic powder $\mathrm{Bi}_{1.6} \mathrm{~Pb}_{0.4} \mathrm{Sr}_{2} \mathrm{Ca}_{2} \mathrm{Cu}_{3} \mathrm{O}_{y}$ was used for electrophoretical deposition and the treatment of the conductors includes repeated cycles of pressing and annealing. In this work texture and structure analysis is accompanied by measuring of the critical current density, and thus, the dependence of current-carrying ability on structure parameters of the ceramic layer could be considered.

\section{INVESTIGATED SAMPLES}

The bismuth ceramic powder of the above indicated composition was produced by cryochemical synthesis and annealed by two different regimes:

regime $\mathrm{A}$ - annealing in air at $830^{\circ} \mathrm{C}$ during 5-6 hours;

regime $\mathrm{B}$ - annealing by regime $\mathrm{A}+$ additional annealing in an argon-oxygen atmosphere at $840^{\circ} \mathrm{C}$ during 24 hours.

Two serieses of samples differing by the annealing regimes of the initial powder were fabricated. Letters A or B are included in the sample number to indicate the initial treatment of the used powder.

The powder was deposited on a silver foil $50 \mu \mathrm{m}$ thick by means of electrophoretic process. The initial thickness of the ceramic coating was approximately $20 \mu \mathrm{m}$. Two coated silver foils were assembled together in such manner that the substrates were outside. This three-layer composite was pressed $(\mathrm{P}=1.5 \mathrm{GPa})$ and then annealed at $830^{\circ} \mathrm{C}$ during 50 hours. The cycle, consisting of pressing and subsequent annealing, 
was repeated up to 5 times. The figure in the sample number indicates how many such cycles were used by its fabricating. The figure " 0 " defines the initial powder of each series.

In order to prepare a sample for X-ray analysis, the three-layer composite was split into two parts by stripping of the silver foil. As a rule, the inner ceramic layer was divided by this procedure into two unequal parts: almost all ceramics remained on one of the two foils while the other foil retained only traces of ceramics. The thickness of the first part was within the limits 5-20 $\mu \mathrm{m}$ depending on the number of cycles. These thicker fragments of the broken composites were investigated. In one case the remainder of the ceramic layer on the stripped foil was analyzed also. In order to mark the corresponding sample the symbol $*$ was used.

\section{MEASURING PROCEDURES}

X-ray analysis of the ceramic layer was carried out by the diffractometers "Rigaku" and DRON-3M using copper and iron radiation. Both standard and modified analytical methods were used.

Phase Analysis. Qualitative phase analysis included computer comparison of the measured spectrum with the data base for known inorganic substances, among which different oxides were of the most interest. In order to estimate the reliability of the results, a reliability factor was calculated taking into account differences of the angular position of the X-ray lines and intensity ratios of the analyzed and standard spectrums.

Quantitative phase analysis of textured materials is connected usually with significant difficulties because of essential intensity redistribution between different lines in the diffraction spectrum. However, in the case of bismuth ceramics the textures of the analyzed phases are similar, as we established earlier (Perlovich et al., 1992) for the phases 2212 and 2201, and this circumstance simplifies the situation. Since textures of different phases retain their mutual similarity by passing from one sample to another, the ratio of analytic lines intensities alters following the relative variation of phase contents for the investigated series of samples.

In order to estimate the phase content in the ceramic sample, ratios of integral intensities of two lines of different phases in spectrums of this sample and of a textureless powder standard are usually being compared. Thus, in order to estimate the quantities of the phases 2212 and 2223, Vasileva et al. (1990) used the line (008) of the phase 2212 and the line $(0010)$ of the phase 2223; at the same time Liang et al. (1990) and Hayashi et al. (1989) used for this purpose the lines (008), (115) and (00 10), (00 12) respectively. In the work by Briggs et al. (1990) calibration curves for mixtures of the powder phases 2212 and 2223 were obtained from integral intensity measurements of the lines (002) of these phases. However, it has to be taken into account that the smaller the Bragg angle, the smaller is the thickness of the reflecting layer. Therefore, data of the mentioned works characterize predominantly the phase composition of different layers depending on the used X-ray lines.

In the present work absolute contents of the phases 2212 and 2223 were determined by use of the calibration curves obtained in the work of Briggs et al. (1990) for the lines (002). It was assumed that this plot is applicable for estimation of phase composition not only in the case of a textureless powder mixture, but also in the case of textured samples when both phases have the same texture. The lines (002) are very 
intensive even in the spectrum of the textureless standard and therefore they are the most suitable for quantitative phase analysis in the case of a homogeneous sample. But since the Bragg angles of these lines are very small, the calculated phase composition characterizes a very thin surface layer only, which, in the initial unbroken composite, was adjacent to the silver substrate. Therefore, in order to obtain data about inner layers of ceramics, X-ray lines at higher Bragg angles were measured corresponding to higher orders of reflection from basal planes, i.e. the line (00 12) for the phase 2212 and the line $(0014)$ for the phase 2223 . The ratio of their integral intensities was used to characterize relative changes of phase composition in the ceramics.

Since for textured ceramic samples these lines are very intensive, by their usage the relative amount of both phases can be determined with a high precision. At the same time these lines can not be used to determine absolute contents of the phases 2212 and 2223 , because their intensities in the spectrum of the textureless specimen are too small. Thus, for the determination of absolute phase contents the lines (002) were used, while the lines $(0012)$ and $(0014)$ were more preferable for looking after small relative changes of the phases. Besides that, alternative usage of different X-ray line pairs allows to compare data, characterizing layers of different thicknesses, and thereby to catch the regular phase inhomogeneity, connected with a nearness of the silver substrate and with its influence on structure formation in the ceramics.

Texture Analysis. The crystallographic texture of the ceramic layer was analyzed by direct pole figures (001), characterizing the spatial distribution of normals to basal planes for grains of the phase 2223. X-ray measuring of direct pole figures (DPF) was realized with an automatic texture set by the specimen tilt method (Borodkina and Spector, 1981). Program control of the texture set was carried out by the computer system KBR. The obtained data were processed by a computer "MERA-60" with special programs.

The plotted DPFs (001) as well as any other (Figure 1) testify, that the ceramic layer of the investigated samples has an axial texture of basal type, allowing to attain the

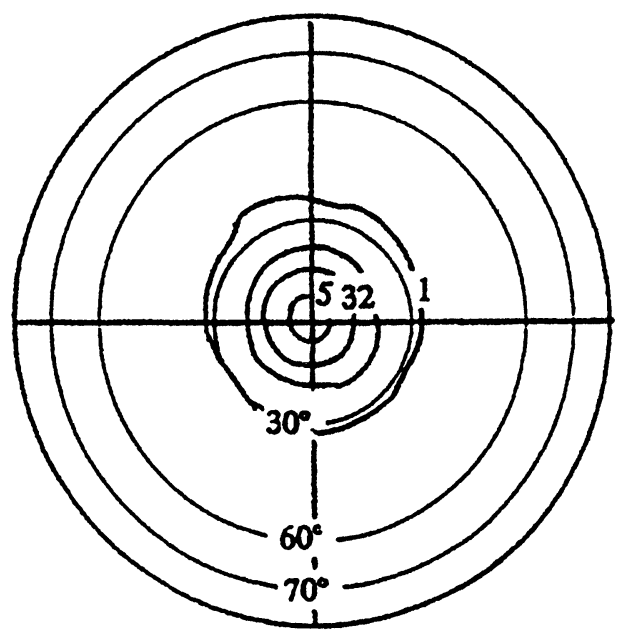

a

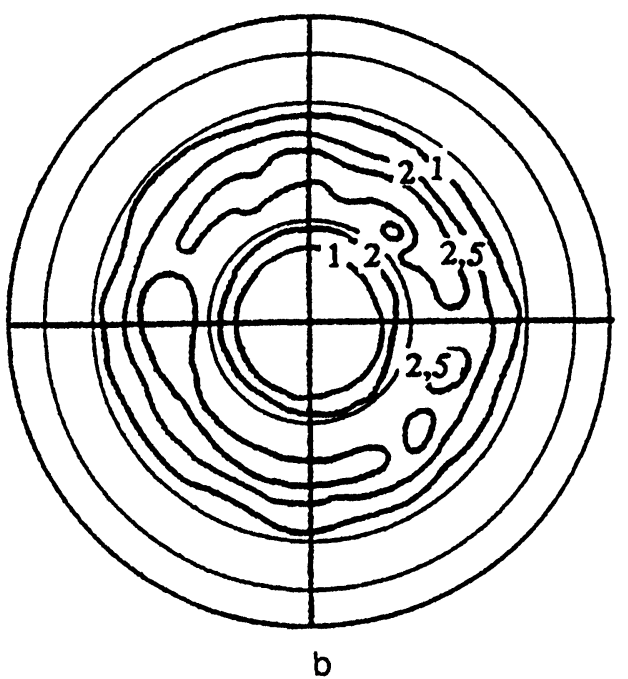

Figure 1 Typical DPFs (001) (a) and (117) (b) for the phase 2223 of bismuth ceramics in threelayer composite. 
maximal density of electrical current in the conductor plane. For quantitative estimation of texture sharpness it is sufficient to consider the distribution of normals [001] in a radial section of DPF (Perlovich et al., 1992). Taking into account the axiality of the observed texture, we limited texture measuring by registration of the specimen tilt curve, which characterizes the variation of the X-ray intensity of the reflection $(00 \mathrm{~L})$ as the tilt angle increases with quick rotation of the sample around the normal to the investigated surface (Figure 2).

The experimental curve having been plotted initially in intensity units was recalculated in pole density units. For this purpose it was approximated by a superposition of Gauss and Cauchy functions followed by extrapolation of the curve over the whole interval of tilt angles. This procedure allowed to make the necessary normalization of the data and to change them into units of the true pole density.

The following parameters of the plotted distribution are estimated:

- the maximal value of pole density for the given curve $\left(P_{o}\right)$;

- the width of the texture maximum on the half of its height $\left(B_{1 / 2}\right)$;

- the volume fraction of grains, whose normals to basal planes deviate from the normal to the investigated surface by no more than $10^{\circ}(\mathrm{W})$.

The critical current density of the fabricated superconductors was measured at $77 \mathrm{~K}$ without magnetic field by the four-point method.

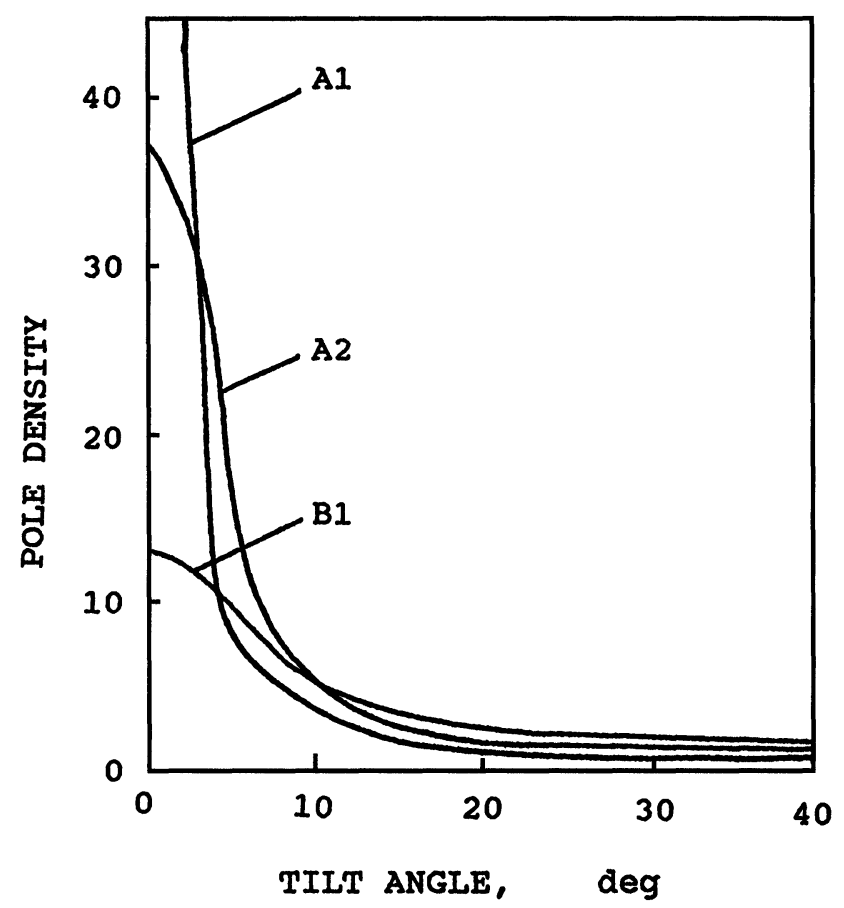

Figure 2 Some radial distributions of basal normals for the ceramic phase 2223 in three-layer composites. 
Table 1 Dependence of current-carrying ability on the structure of the ceramic layer in composite superconductors.

\begin{tabular}{|c|c|c|c|c|c|c|c|}
\hline $\begin{array}{l}\text { Sample } \\
N\end{array}$ & $\begin{array}{l}I_{2212}^{002} \\
I_{2223}^{002}\end{array}$ & $\begin{array}{l}I_{2212}^{0012} \\
I_{2223}^{0014}\end{array}$ & $\begin{array}{c}V_{2223}^{*} \\
\text { [mass \%] }\end{array}$ & $P_{o}$ & $\begin{array}{c}B_{1 / 2} \\
\text { [degree] }\end{array}$ & $\begin{array}{c}W \\
{[\%]}\end{array}$ & $\begin{array}{c}j_{c} \\
{\left[10^{3} \mathrm{~A} / \mathrm{cm}^{2}\right]} \\
(77 \mathrm{~K}, 0 T 1)\end{array}$ \\
\hline A0 & $\infty$ & $\infty$ & 0 & - & - & - & - \\
\hline $\mathrm{A} 1$ & 0.49 & 0.75 & 74 & 119 & 1.8 & 61 & 0.1 \\
\hline $\mathrm{A} 1 *$ & 0.55 & - & 70 & - & - & - & - \\
\hline A2 & 0.09 & 0.10 & 95 & 37 & 4.0 & 37 & 1.1 \\
\hline A3 & 0.07 & 0.02 & 96 & 25 & 4.8 & 34 & 1.6 \\
\hline A4 & 0.06 & 0.17 & 96 & 17 & 5.6 & 26 & 1.1 \\
\hline A5 & 0.03 & 0.10 & 100 & 12 & 6.4 & 20 & 0.6 \\
\hline B0 & 0.14 & 1.12 & 76 & - & - & - & - \\
\hline B1 & 0 & 0.05 & 100 & 13 & 7.4 & 24 & 1.9 \\
\hline B2 & 0 & 0.08 & 100 & 14 & 8.0 & 27 & 2.5 \\
\hline B3 & 0 & 0.05 & 100 & 17 & 7.3 & 31 & 3.8 \\
\hline B4 & 0 & 0.05 & 100 & 16 & 7.8 & 30 & 4.0 \\
\hline B5 & 0 & 0.03 & 100 & 19 & 7.1 & 35 & 4.2 \\
\hline
\end{tabular}

* - estimation of the relative content of the phase 2223 in the ceramic layer was carried out in accordance with the calibration plot (Briggs et al., 1990) and by the intensity ratio of X-ray lines (002) for both phases.

\section{EXPERIMENTAL RESULTS AND THEIR DISCUSSION.}

Measured or calculated values of critical current density $j_{c}$, ratio of X-ray line intensities, content $\mathrm{V}$ of the phase 2223, and different texture parameters for the investigated samples are presented in the Table 1.

According to the results of X-ray phase analysis, in all investigated samples, except sample $A 0$, the phase 2223 is predominant, while phase 2212 is present in smaller quantity and phase 2201 was not revealed at all. Along with the main phases 2223 and 2212, oxides $\mathrm{CuO}, \mathrm{CuO}_{2}, \mathrm{Bi}_{2} \mathrm{O}_{3}, \mathrm{Ca}_{2} \mathrm{PbO}_{4}$ and $\mathrm{Sr}_{2} \mathrm{CuO}_{3}$ can be present in the ceramics also. They were identified unambiguously in the powder of the first series (A0). Quantitative estimation of oxide phase content was not carried out because of impossibility to take into account texture coefficients for each phase. Obviously, the content of oxide phases correlates with the relative content of the phase 2212 since the initial mixture of powders corresponded to the stoichiometric composition 2223 and oxide phases account for the residual fraction of metal components which did not get into the phase 2212.

The difference of phase composition between samples of serieses A and B is caused by the corresponding difference between powders used for their fabrication. The annealed powder (A0) consisted predominantly of the phase 2212 and accompanying oxide phases, while subsequent annealing of this powder at $840^{\circ} \mathrm{C}$ promoted the formation of the phase 2223 (B0). Intensities ratios of X-ray lines (002) of the phases 2212 and 2223 show, that the samples A2-A5 and B1-B5 are single-phased practically with an error of $\pm 5 \%$. However, if their phase content is analyzed by X-ray lines $(00$ 12 ) and (00 14), characterizing the ceramic layer on the whole, in each series a decrease of the phase 2212 can be noticed as the total annealing time increases, altering with periodic additional pressing of the three-layer composite. Monotonous increase of 
the phase 2223 depending on the duration of annealing at $830^{\circ} \mathrm{C}$ is caused by a redistribution of metal ions by solid-state diffusion (Shneider et al., 1993).

The texture of the ceramic layer was estimated by use of texture parameters for the predominant phase 2223. Comparison of texture perfection in the phases 2212 and 2223 was carried out only for the sample A1, in which the content of the phase 2212 was maximal. Obtained results confirm mutual correlation of texture parameters for both phases being present in the ceramic layer. The analogous conclusion was made earlier (Perlovich et al., 1992) concerning textures of the phases 2201 and 2212. Texture similarity of the phases 2212 and 2223 indicates that the processes of texture formation in these phases are interrelated.

In the course of the electrophoretic deposition a packing texture of plate-like powder particles forms, while the perfection of this texture depends on the particle size distribution of powder and the regime of the electrophoretic process as we stated earlier (Isaenkova et al., 1992). Pressing causes corrective rotation of powder particles and breakage of particles with unfavourable orientations, promoting further perfection of the basal type texture. If the ceramic layer is partially remelted (Perlovich et al., 1992), annealing amplifies its initial texture owing to essentially facilitating reorientation of plate-like particles in the liquid phase parallel to the foil plane and their following anisotropic growth during decreasing temperature.

In this work the analysis of texture data relied in the first place on the integral characteristic $\mathrm{W}$ of the distribution of basal normals. The mutual correspondence between the half-width of the texture maximum and volume fraction of crystallites forming this maximum is not always observed (for example compare samples A5 and A1). This fact is connected with different characters of the profile of the texture maximum. Thus, for the sample A5, the relatively small width of the texture maximum is accompanied by a low value of the parameter $\mathrm{W}$, i.e. the sharp texture maximum forms on the background of a significant textureless component.

Therefore the texture of the ceramic layer in the sample A5 should be considered as two-component: the first component consists of grains which retained their basal orientation during the five-cycle treatment and the second component includes grains with new random orientations. It is obvious that these latter grains grew from the melt while having small fractured fragments of initial particles as internal nuclei.

Consideration of the obtained data shows that the texture perfection of the phase 2223 depends on the content of the phase 2212. As its content decreases in consequence of annealing, the basal texture of the phase 2223 becomes weaker. Hence, the initial phase 2212 is responsible for the formation of the basal texture, while the derivative phase 2223 only inherits its texture and neither develops nor retains it without controlling influence of the phase 2212. The mechanism of basal texture formation, proposed by Perlovich et al. (1992), takes place in the phase 2212 predominantly. As to the phase 2223 , the mechanisms of basal texture inheritage and scattering are of further interest.

If the phase 2223 forms owing to solid-state diffusion of metal ions into the crystalline lattice of the initial textured phase 2212, the texture inheritage is natural. But when the phase 2223 arises by crystallization of the melt, different texture variants are possible. Partial melting of the ceramics can have two different effects on further texture development. Due to forces of surface tension the pressing package of coarse platelike particles improves. As a result, the basal texture component, corresponding to these particles, sharpens in both phases. But the effect of basal texture sharpening in the phase 2223 is a consequence only of the analogous effect, which takes place originally 
in the phase 2212. Would new grains of the phase 2223 form either inside of the initial plate-like particles by diffusion or on their surface by crystallization of the melt, the texture of the phase 2223 would copy the basal texture of the phase 2212 , prevailing in these particles at first.

At the same time, under partial melting of the ceramics, small edge fragments of irregular shapes separate from plate-like particles of the initial powder, lose their basal orientation in the melt between particles and become nuclei for new randomly oriented grains of the phase 2223 during the following crystallization of the melt. Depending on the relative volume of the melt and on the fraction of the phase 2223 in platelike powder particles, the latter or the former effect can prevail in the process of texture formation under annealing of the composite, leading either to perfecting or to scattering of the basal texture.

According to the data presented in the table, the texture perfection of the ceramic layer in samples of the series A decreases as the total annealing increases. For samples of the series B some slight texture perfection can be noticed at the same time. Figure 3 demonstrates different monotonous changes of the texture parameter $\mathrm{W}$ depending on the annealing time for both serieses of samples. These opposite tendencies in changing of texture sharpness could be predicted on the basis of the above described model.

Since in samples of the series A the significant fraction of easy-melting oxides intensifies partial melting of the ceramics at the used annealing temperature (Shneider et al., 1993; Oskina et al., 1992; Kijima et al., 1988; Bykov et al., 1993),

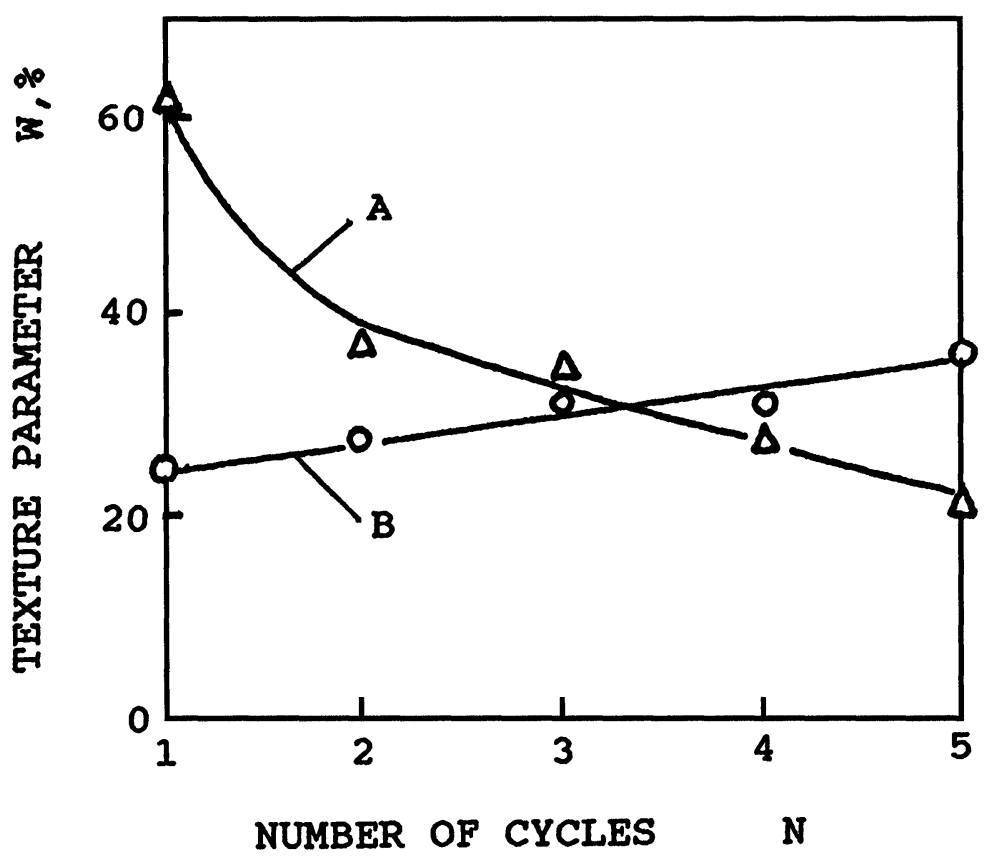

Figure 3 Influence of cyclic treatment (pressing + annealing) of three-layer composite on texture perfection of the ceramic phase 2223. Experimental points for samples of serieses A and B are shown with different signs ( $\Delta$ and $o$, respectively). 
the conditions develop there for emergence of numerous small fragments separated from initial particles due to local melting of inner oxide inclusions. In relatively spacious cavities between plate-like particles these fragments lose the basal orientation, causing afterwards a noticeable texture scattering in the phase 2223 by melt crystallization.

In samples of the series B the effect of partial melting is weak because of the initial low content of the phase 2212 as well as of accompanying oxide phases. Therefore by treatment of these samples, all considered processes, connected with melting of ceramics, can not develop, though some texture perfection is caused by corrective rotation of plate-like particles under pressing. As a result, for the series B the value of the parameter W (24-35\%) corresponds to its level in ceramic layers fabricated by pressing or rolling without solid-phase sintering.

In Figure 4 a graph is represented showing the dependence of critical current density on phase composition of the ceramic layer for the investigated composites. As the relative content of the phase 2212 decreases down to zero, the critical current density grows, and this tendency can be observed for both serieses of samples.

The dependences of current density on the texture parameter $\mathrm{W}$, being shown in Figure 5 for both serieses of samples, are linear for values of this parameter up to $30-35 \%$. By further perfection of the basal texture in samples of the series A the current density passes through a maximum and then decreases. This testifies that in reality the dependence of the current density on the texture perfection has a complex character and that an excess, above some optimal texture perfection, influences the superconducting properties of the composite negatively. At the same texture perfection, higher values of current density for samples of the series B are caused by larger contents of the phase 2223, which is found to be the most favourable for superconductivity with reference to the used ceramics.

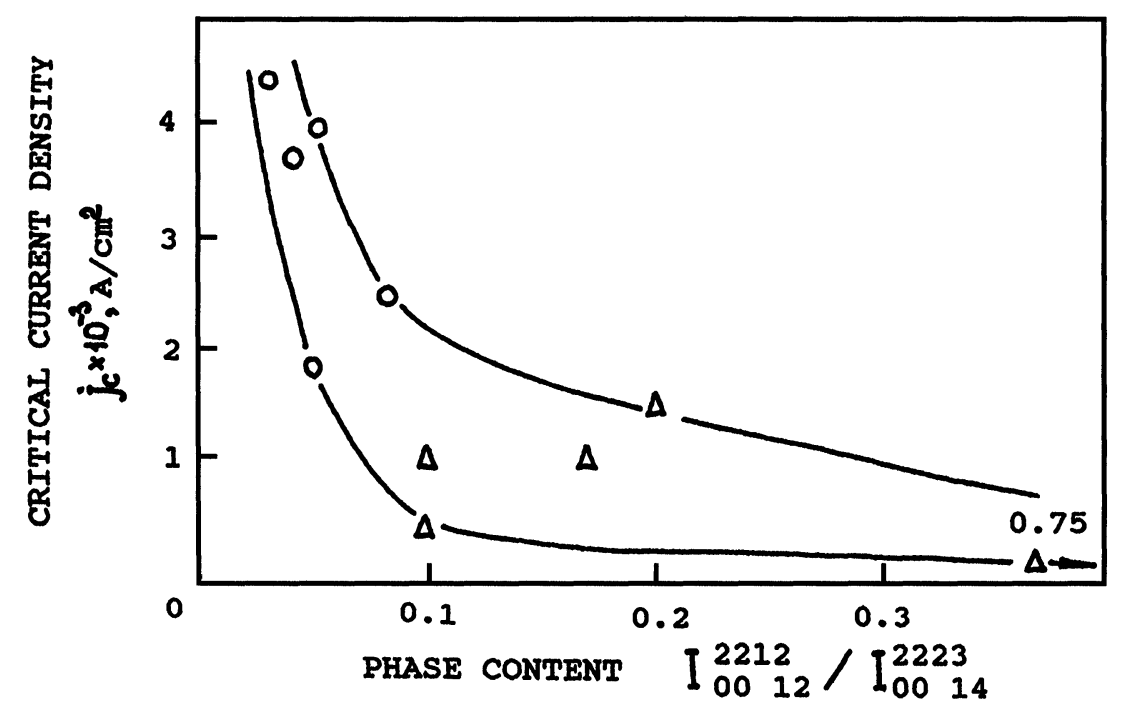

Figure 4 Variation of critical current density $j_{c}$ depending on the relative content of the phases 2212 and 2223. Two curves illustrate the bandwidth of the relationship between phase content and critical current density. 


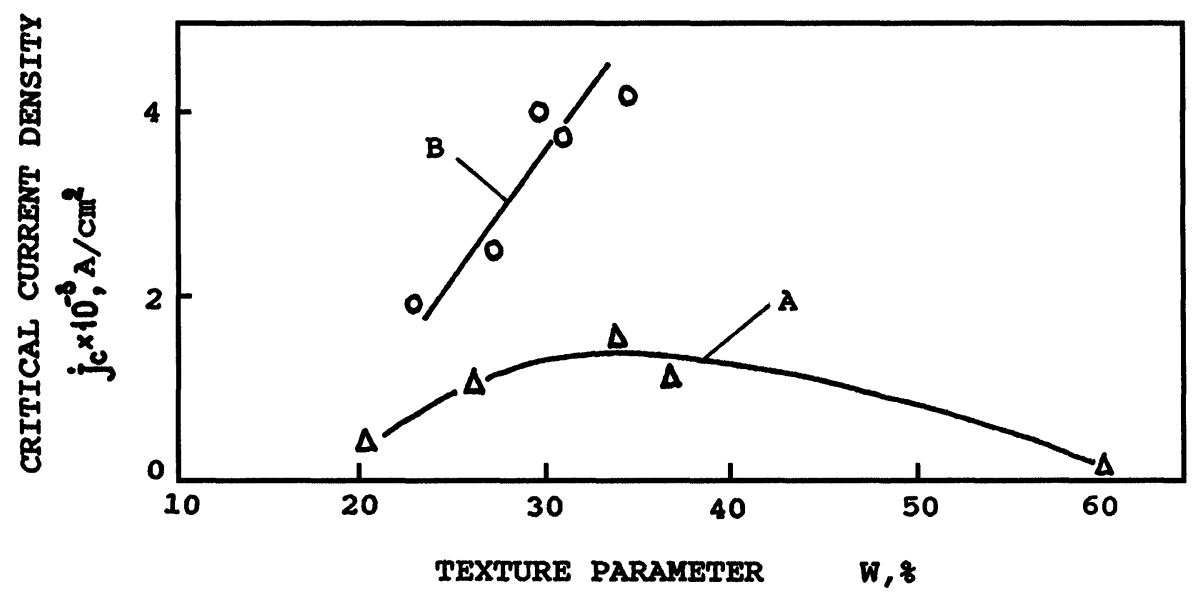

Figure 5 Dependence of critical current density $j_{c}$ on texture perfection, estimated by the fraction $\mathrm{W}$ of basic normals deviating from the normal to the sample surface by no more than $10^{\circ}$. Marking of experimental points is the same as on Figure 3.

As the texture becomes more perfect, mutual misorientation of grains within the sample decreases. In the case of the axial basal texture its perfection characterizes the mutual misorientation of basal planes. The higher the texture parameter $\mathrm{W}$, the lower is the average misorientation of basal planes at boundaries between neighbouring grains. If we observe a current density decrease by an excess of texture perfection above some degree, with other conditions being equal, it should be concluded that the change of the character of intergranular boundaries is responsible for this decrease.

Thus, for attainment of increased current density in a sample consisting of the phase 2223 predominantly, the presence of intergranular boundaries with some optimal misorientation of basal planes is necessary. According to texture data, this optimum is equal to several degrees. Comparison of data for samples with different phase contents shows that interphase boundaries do not influence the superconductivity in the same manner as intergranular boundaries. Apparently, the mechanism of this influence is connected with pinning.

The considered regularities are reflected most obviously in the diagram, where current density is represented as the function of both mentioned structure characteristics, simultaneously (Figure 6). In the diagram the distribution of critical current density $j_{c}$ for three-layer conductors depending on phase composition (x-coordinate) and basic texture perfection (y-coordinate) is shown. The region can be localized, in which values of the current density are of practical interest. This region is adjacent to the y-axis, being restricted by $20 \%$ - and $40 \%$-levels of the parameter $\mathrm{W}$. The diagram is convenient for revealing optimal fabrication regimes with reference to composites of the investigated type.

According to this diagram, further increase of current density in composites on the basis of the bismuth ceramics can be obtained by fitting of the regimes for pressing and heat treatment which would produce the optimal combination of structure parameters in ceramics. By this way the critical current density for three-layer composite of the investigated type was increased up to $7 \cdot 10^{3} \mathrm{~A} / \mathrm{cm}^{2}(77 \mathrm{~K}, 0 \mathrm{~T} 1)$. 


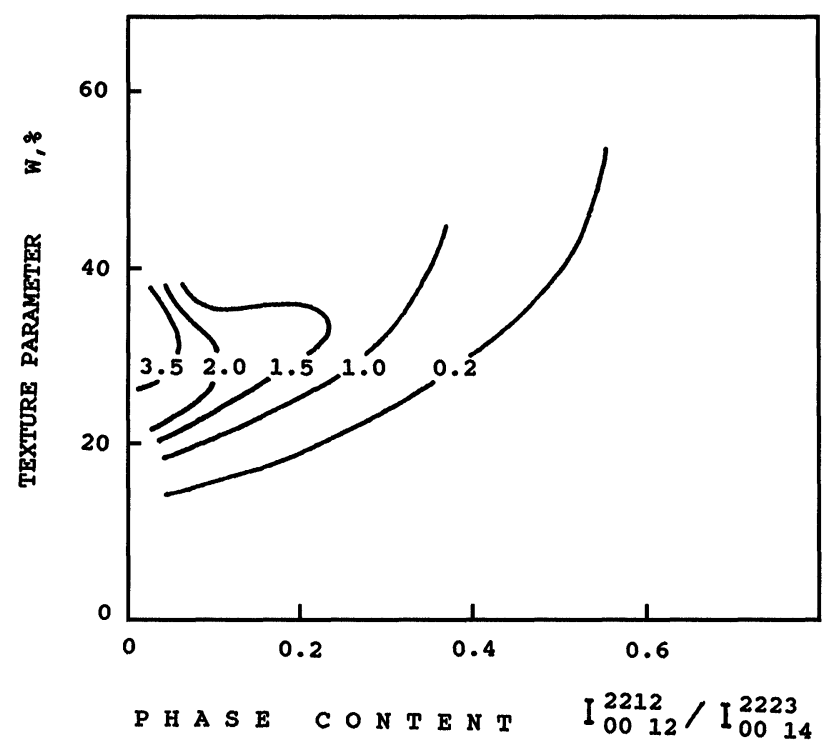

Figure 6 Diagram of critical current density for three-layer conductors depending on their phase composition and texture perfection. Values of critical current density are indicated at corresponding contours.

\section{CONCLUSIONS}

1. Phase changes and texture formation are studied for ceramics of composition $\mathrm{Bi}_{1.6} \mathrm{~Pb}_{0.4} \mathrm{Ca}_{2} \mathrm{Sr}_{2} \mathrm{Cu}_{3} \mathrm{O}_{x}$ in three-layer composite conductors Ag-HTSC-Ag produced by electrophoretic deposition. Depending on phase composition in the initial ceramic powder, cyclic treatment of the composites, including pressing and annealing at $830^{\circ} \mathrm{C}$, causes different tendencies in the development of the basal texture, being necessary for attainment of high critical current density.

2. The basal texture forms primarily in the phase 2212 , which prevailed in the initial ceramic powder and then transforms into the phase 2223 gradually during heat treatment. The derivative phase 2223 , being of practical interest, shows either heritage or scattering of the basal texture in accord with the liquid phase content in the sample under annealing.

3. When the phase 2223 forms by solid-state diffusion and melt crystallization on initial aligned particles, the basal texture is inherited. Crystallization of the phase 2223 on small fragments, separated from plate-like aligned particles in the melt, results in partial scattering of the basal texture.

4. The larger the content of the phase 2223 in the ceramics, the higher is the critical current density. Rise of the texture perfection parameter W up to $40 \%$ assures a gain in critical current density also, while further texture perfection causes already some decrease of current density. The latter effect testifies that it is necessary to retain some amount of intergranular boundaries with the optimal misorientation in the phase 2223 for supporting of superconductivity. 
5. A diagram was constructed for composites Ag-HTSC-Ag, connecting their phase composition and texture perfection with the measured critical current density. The diagram can be used to control the processes of ceramics treatment for superconductor fabrication.

\section{Bibliography}

Borodkina M. and Spector E. (1981). X-ray Analysis of Texture in Metals and Alloys/Metallurgia, 271 p. (in Russian).

Briggs A., Bellami B., Denton I. and Perks J. (1990) Preparation of single phase bismuth-based 2212 and 2223 superconducting oxides. J. Less-Common Metals, 164/165, 559-567.

Bykov A., Kucheiko S., Kosynkin V., Shatalov V., Kutepov V., Mitrokhin V. and Radchenko I. (1993). The influence of the carbon percentage in 2223 on the critical current. Sverkhprovodimost' (KIAE), 6(7), 1463-1469 (in Russian).

Hayashi Y., Kogure H. and Gondo Y. (1989). Growth of the high-T $\mathrm{T}_{c}$ phase in the Bi-Pb-Sr-Ca-Cu-O superconducting oxide system. Jpn. J. Appl. Phys., 28(12), L2182-L2184.

Isaenkova M., Perlovich Yu., Kalinin N. and Gorshkov T. (1992). Texture formation in bismuth ceramics under pressure treatment. In Proceedings of 13th International Conference "Strength and Plasticity Physics of Metals and Alloys", Samara (Russia), p. 167 (in Russian).

Kijima N., Endo H. and Tsuchiya I. (1988) Reaction mechanism of forming the high- $\mathrm{T}_{c}$ superconductor in the Pb-Bi-Sr-Ca-Cu-O system. Jpn. J. Appl. Phys., 27(10), L1852-L1855.

Liang R., Ishii H. and Kawaji H. (1990) Synthesis of superconducting 2223 in the lead-added BiSr-Ca-Cu-O system. Jpn. J Appl. Phys., 29(8), L1412-L1414.

Oskina T., Kazin P., Tretyakov Yu., Kozlovskiy V. and Lapshina I. (1992) Synthesis of superconducting copper oxides by preliminary annealing of precursors. Sverkhprovodimost' (KIAE), 5(7), 1298-1305 (in Russian).

Perlovich Yu., Isaenkova M., Nikulin A., Shikov A., Kalinin N. and Gorshkov T. (1992) Structure and texture of electrophoretically deposited bismuth ceramic films determined from X-ray diffraction analysis. Sverkhprovodimost' (KIAE), 5(4), 748-756 (in Russian).

Shneider A., Bulishev Yu., Selyavko A. and Serich S. (1993). Phase equilibrium and crystal growth in Bi-Sr-Ca-Cu-O system. Sverkhprovodimost' (KIAE), 6(1), 136-143 (in Russian).

Vasileva I., Reddy B., Boldireva N., Kuropyatnik I., Romanenko A. and Malahov V. (1990) Effect of system Bi-Sr-Ca-Cu-O alloying by aluminium and silver. Sverkhprovodimost' (KIAE), 3(11), 2608-2615 (1990) (in Russian). 